\title{
REAL-TIME HEAD POSE ESTIMATION WITH SVM MODEL FOR FRONTAL FACE CLASSIFICATION
}

\author{
Loubrys L. Rojas Reinoso, Fernando L. Gutiérrez López, José C. Gutiérrez, Graça Bressan \\ and Wilson Vicente Ruggiero \\ Laboratório de Arquitetura e Redes de Computadores (LARC), Escola Politécnica da Universidade de São Paulo, Brazil
}

\begin{abstract}
Head pose estimation (HPE) has been widely studied in the last years due to its many applications in face analysis systems. The use of such systems ranges from the analysis of focus of attention, social interactions or the use in mobile applications in the realization of the currently popular facial animations and / or in face recognition process, where the frontal faces are especially important. Many approaches were proposed focusing mainly in Random Forests and Convolutional Neural Networks (CNN). In this paper, a framework for estimation of the head pose was proposed computing the degrees of freedom (DOF) of the human head using 2D images data only. The framework implements some computer vision algorithms available in publicly machine learning libraries such as OpenCV and Dlib, which allows easy application and re-implementation. In addition, a Support Vector Machine (SVM) model with Radial Basis Function (RBF) kernel was developed for frontal face classification. Experiments conducted on 2D image datasets in constrained environment show that the approach is capable of real-time performance. Were designed three protocols of experiments with two databases for testing the SVM model. Values of $100 \%$ and $98 \%$ for precision and recall, respectively, were achieved classifying frontal faces. Significant results were obtained measuring yaw rotation with 4.24 of mean absolute error for frontal face.
\end{abstract}

\section{KEYWORDS}

Degree of Freedom of Human Head (DOF), Head Pose Estimation (HPE), Support Vector Machine (SVM), Frontal Face Classification, Machine Learning, Computer Vision

\section{INTRODUCTION}

Detection and analysis of faces have been huge problems in computer vision and have been actively researched for many years. Despite the progress made in recent years in face detection, estimating the position of the head remains a challenging task, due to the variety of head expressions, races, gender, and other challenges. The interest of the research community in HPE is mainly due to a large number of applications that require or are improved by a reliable HPE system: face recognition, liveness detection, facial animation, human-computer interaction, people behavior understanding, driver assistance in automotive field and so on (Borghi et al., 2018). Researchers see the HPE task as identifying the orientation of the head of a person related to the view of the camera. Many machine learning algorithms have been proposed to address this difficult problem, focusing mainly in Random Forest (Fanelli et al., 2011; Valle et al., 2016), Convolutional Neural Networks (CNN) (Ranjan, Patel and Chellappa, 2017; Borghi et al., 2018) and landmark-based methods (Baltrusaitis, Robinson and Morency, 2016; Bertók and Fazekas, 2016), which use facial landmarks to estimate the head pose. Landmark-based approaches take the advantage of the geometry of facial features (Bertók and Fazekas, 2016).

In this paper, we present an end-to-end system capable to detect a face and its landmarks, if exit, in a given image and define if the head is frontal or not to the camera. To determine the pose, will be located the landmarks of the face and solved the Perspective n-Point (PnP) problem; consequently, the DOF of the human head will be extracted, which will inform us of the position of the head with respect to the camera. Subsequently, an SVM model will be trained with the extracted degrees, learning to classify whether a face is frontal or not to camera. The core intention of the developed approach is to have good performance for real-time applications. 


\section{HEAD POSE ESTIMATION APPROACH}

The proposed approach is also capable of detecting the face and its landmarks, before performing HPE task. Due the approach estimates the pose of the head using only single 2D images, the task will be done based on the 2D projection of a 3D head, but first we need to detect a face and several feature points from it.

Face detection, landmarks detection, and HPE have often been studied in the literature as separate problems. Many papers have related the benefits of working with correlated tasks. With the publicly and available library Dlib (King, 2009), face and landmark detection can be performed. After a 2D image has been acquired from different kinds of media, the system will proceed to detect and localize faces in the given image and will return the number and the sizes, if exit any face on the image. Dlib face detector is made using the classic Histogram of Oriented Gradients (HOG) (Dalal and Triggs, 2005) features widely used in the literature for face and pedestrian detection (Wang, Han and Yan, 2009; Rekha and Kurian, 2014). After face detection, we need to find several points from the face. To compute that, the $68(\mathrm{x}, \mathrm{y})$-coordinates that map the facial structures on the face inside the Dlib library, was used. The model used by Dlib was trained with 68 points annotations of the iBUG 300-W dataset (Sagonas et al., 2013). These facial landmarks will be helpful to calculate the DOF.

In computer vision, the pose of an object refers to its relative orientation and position with respect to a camera. Our HPE method is based on the solution of Perspective n-Point problem (PnP problem). In this problem the goal is to find the pose of an object when we have a calibrated camera, and we know the locations of some 3D points on the object and the corresponding 2D projections in the image. To calculate 3D pose of an object in an image we need the 2D coordinates of a few points and 3D locations of the same points. The 2D coordinates of the points are resulted of the facial landmarks detection performed before, they define the camera coordinates system. We also have the same number of 3D model points in appropriate model coordinate system. Ideally, a 3D model of the person head on the image is necessary to get 3D locations of the points, but in practice it is not, just 3D locations of these points in some arbitrary reference frame are needed.

The 3D locations of facial features are in world coordinates. Knowing the translation and rotation we could transform these points in world coordinates in camera coordinates. In camera coordinates these points can be projected onto image plane using the intrinsic parameters of the camera (focal length, optical center and radial distortion). All this can be done using Equation 1, where $A$ is the camera matrix which contains the intrinsic parameters and principal points. The role of $A$ is to project $3 \mathrm{D}$ points of camera coordinate onto $2 \mathrm{D}$ image plane, and $[R \mid t]$, is the joint rotation-translation matrix whose transforms points from the $3 \mathrm{D}$ world coordinate system to $3 \mathrm{D}$ camera coordinate system.

$$
p_{i}=A[R \mid t] P_{i}
$$

Equation 1

For all of these experiments, we assume that camera is calibrated due their intrinsic parameters are not always known; then zero lens distortion is assumed, and run iterative method based on Levenberg-Marquardt optimization to find 2D-3D correspondence, which is implemented as the function SolvePnP in OpenCV library (Bradski and Kaehler, 2008). SolvePnP implements several algorithms for pose estimation which can be selected using the parameter flag. By default, it uses the flag SOLVEPNP_ITERATIVE which is essentially the Direct Linear Transform (DLT) solution followed by Levenberg-Marquardt optimization. When the estimated pose is perfect, the 3D points projected onto the image plane will line up almost perfectly with the $2 \mathrm{D}$ facial features.

One of the benefits for performing HPE solving PnP problem with OpenCV library is obtaining the DOF or euler angles. With these angles a Support Vector Machine (SVM) model was trained to classify faces in frontal or non-frontal. SVM (Hearst et al., 1998) are a particularly powerful and flexible class of supervised algorithms for both classification and regression. SVM finds an optimal hyperplane which helps in classifying new data points. Due to the characteristic of the data analyzed, the more appropriate kernel trick will be the radial basis function (RBF). It can map an input space in infinite-dimensional space. Tuning the parameters of the SVM could be a hard task. A good solution could be the implementation of GridSearchCV. It is a function of the Scikit-learn library (Pedregosa et al., 2011). This function will allow defining the best parameters for the SVM. Various values of $C$ and gamma, and the desired kernel will be passed into the function, and later should obtain the best parameter combination. 
An SVM model will be trained to separate frontal from non-frontal. Frontal faces will be separated from non-frontal ones for training. This supervised learning will show to the classifier the behavior of the DOF (yaw, pitch, roll) in frontal and non-frontal faces. The measurement of the three angles should be near to 0 degrees in frontal faces. The SVM model training and the selection of different parameters will be done using the Scikit-learn library. The performance of the SVM classifier depends a lot of the images in which it was trained, so training the classifier with the proper databases is a very important phase. To train the model the following two databases were chosen: FaceDB Database (Peer et al., 2014) and FEI Face Database (Eduardo Thomaz and Antonio Giraldi, 2010). These banks were created under controlled conditions, designed among other purposes, for training and testing face detectors, under different illumination conditions and facial expressions and positions. Brief description of the composition of the databases is given below:

- FaceDB Database: database with approximately 800 images created by the University of Ljubljana. Images from 114 people were used, $90 \%$ men. The photos were taken under uniform lighting conditions and with a white background. All images have a resolution of $640 * 480$ pixels and were taken with the same camera. The database was designed for face detection and recognition, feature detection and 3D face modeling.

- FEI Face Database: is a database of 2800 images from 200 people, half men, half women; created by the FEI Artificial Intelligence Laboratory in São Bernardo do Campo, São Paulo, Brazil. The photos have a homogeneous white background and vertical front position, with profile rotation of up to 180 degrees.

\section{PARTIAL RESULTS}

The results in the research were obtained with the following hardware: Intel (R) Core i7-7500U CPU 2.90 GHz computer with 16 GB of RAM, no GPU assistance, on a 64-bit Windows 10 operating system under the Python programming language. Under this hardware the system works at 40 frames per second, which makes it feasible for application in real-time systems. Three experiments were performed looking to get the best possible classifier, results are shown in Table 1. In Protocol 1, the classifier was trained with the FaceDB Database and tested in FEI Face Database, then, in Protocol 2, the inverse process was performed to analyze behavior variation of the classifier, and finally, Protocol 3 mixed the two databases trying to obtain greater robustness in the SVM model.

With the Scikit-learn library, in addition to training and generating an SVM model, it will also allow evaluating the model through the confusion matrix and the classification report. These will be our metrics to measure the accuracy of our model. The accuracy of the classifier is given by the metrics: Precision, Recall and F1-score. Precision is the percentage of what our model classified as frontal or non-frontal and got it right. The Recall is all that was frontal or non-frontal, the percentage he could identify. F1-score is the harmonic mean of precision and recall, where an F1 score reaches its best value at 1 . This library can also measure the Mean Absolute Error (MAE) which is the difference between the measured value and "true" value.

Executing Protocol 1, it is shown how the model despite being trained with just hundreds of images and low variation in face position angles $(-90,-45,0,45,90)$, it can perform well. Despite having an overall accuracy of $92 \%$, the most critical aspect is the $10 \%$ of images that the system classified as frontal but were not (false positives). The result is given by the low number of images and few angles variation for training before reported. In some scenarios or systems, reducing the number of false positives is very important, due in most cases it defines the level of security of the system. When roles were reversed in Protocol 2, and the training has been performed with FEI Face Database, the results are greatly improved, due the model is trained with a larger number of images and variations in angles, which provides more information on training and thus obtaining a more robust model. In this case, the model eliminates false positives, it classifies as frontal images previously classified in the same way. The accuracy of the model in this training is only affected by a small group of frontal images that the system misclassified. Many images in the FaceDB Database have different facial expressions, aspects that affected the classification. 
Table 1. Results obtained in the different protocols performed

\begin{tabular}{lccccccccc}
\hline & \multicolumn{1}{c}{ Metrics } \\
\cline { 2 - 11 } & \multicolumn{3}{c}{ Precision } & \multicolumn{1}{c}{ Recall } & \multicolumn{3}{c}{ F1-score } \\
\hline Protocols & 1 & 2 & 3 & 1 & 2 & 3 & 1 & 2 & 3 \\
\hline Not Frontal & 0.96 & 0.97 & 0.96 & 0.91 & 1.00 & 0.97 & 0.93 & 0.98 & 0.96 \\
Frontal & 0.90 & 1.00 & 0.97 & 0.95 & 0.98 & 0.96 & 0.92 & 0.99 & 0.97 \\
\hline
\end{tabular}

In previous experiments, training and validation were done in one database and testing in the other. In Protocol 3 both databases were used for training and testing, the total images were divided into training, validation, and testing. The unified images bank was divided into $80 \%$ for training and validation, and the remainder for testing. On this occasion, the results show a general accuracy of the model of $97 \%$, but with only $3 \%$ of false positives, which is considered very favorable for a lightweight and not very complex system.

Analyzing the different experiments performed before, can be seen how small databases, such as FaceDB, could be only suitable for testing. Despite the system show a good performance when was trained with a database of hundreds of images and low variety of poses, results suffered a good increase when the system was trained with thousands of images and good range of variety of poses, achieving notable values of 1.00 and 0.98 for precision and recall, respectively, in Protocol 2. The mix of databases could not obtain the best performance of the system due a group of misclassified images were obtained, most of these misclassified images were again faces with non-neutral facial expressions, showing a sensibility of the system to this aspect.

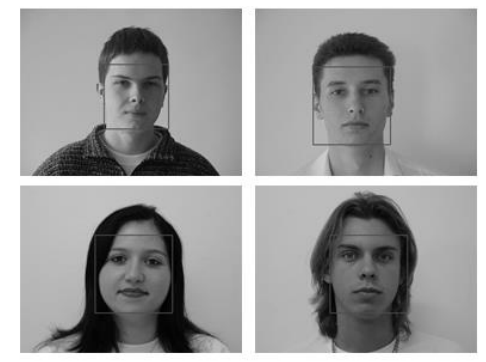

a)

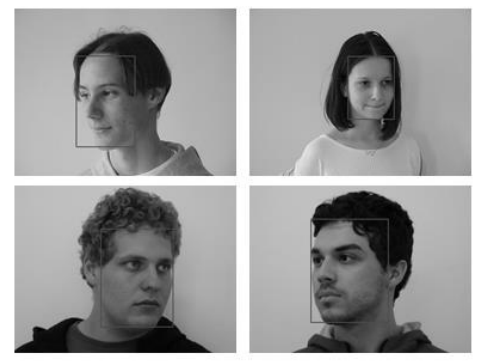

b)

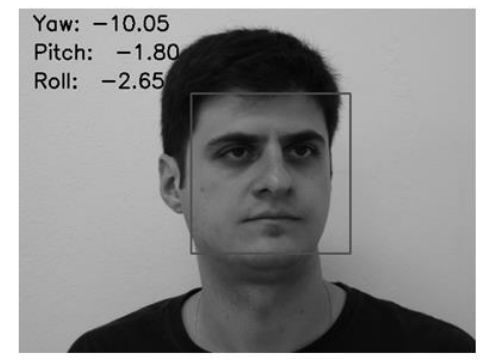

c)

Figure 1. Head pose estimation approach performance. Top: images extracted from FaceDB. Bottom: images extracted from FEI Face Database. a) images classified as frontal by the SVM model. b) images classified as non-frontal by the SVM model. c) examples of extraction of the degrees of freedom (DOF) of human head

Figure 1 shows the results obtained by the proposal classifying faces in frontal or non-frontal and extracting euler angles. Some of the results obtained is a MAE of 4.24 for yaw detection, outperforming the approach presented by (Valle et al., 2016) with a MAE of 7.84 for yaw detection in a laboratory conditions database, and and getting closer to the state-of-the-art. Approaches with better results frequently use Convolutional Neural Networks (CNN) (Borghi et al., 2018; Ruiz, Chong and Rehg, 2018; Yang et al., 2019). The use of deep networks report advantages compared to landmark-to-pose methods, but also limitations. Two of the approaches before mentioned do not report real-time performance. Borghi et al. (2018) approach can work in real-time but needs 3D images acquisition media. Use RGBD (depth) cameras can accurate HPE, but they can be difficult to use outdoors; depth cameras draw more power than RGB and RGBD are higher than for RGB, increasing storage and data transfer times (Ruiz, Chong and Rehg, 2018), all these aspect limit the applicability of this kind of system. The benefits of our proposal are it does not need a 3D image acquisition media, the use of publicly and available ML libraries allow to get a system with simple architecture and easily application and re-implementation in different scenarios and programming languages, and it can also work in real-time without losing precision. The proposal can be easily configurable to give feedback or instructions to the user in the image acquisition process. 


\section{CONCLUSION}

In this paper, a lightweight and simple architecture for HPE was presented. We use the benefits to performance face and landmark detection for HPE. With the help of facial landmarks and solving the PnP problem, the DOF of human head were extracted. An SVM model was generated to classify if a face frontal or not according to the behavior of the DOF. All the system was developed with publicly available machine learning libraries for easy application and re-implementation in different platforms and programming languages. There were obtained as main results: 4.24 of MAE, $100 \%$ and $98 \%$ of precision and recall of our SVM model and speed of $40 \mathrm{fps}$, feasible for real-time application. Due its simple architecture, its accuracy and its ability to perform in real-time, the proposed system could be included in others face analysis systems for social interaction, focus of attention and son on. Results obtained so far as part of a work in progress, so future works will be focused on training and testing the approach in unconstrained environments datasets, analyze how facial expressions could affect the accuracy of this kind of HPE approaches and analyze the benefits of the use of HPE systems in others face related systems such as: face recognition, facial animation and more.

\section{REFERENCES}

Baltrusaitis, T. et al, 2016, 'OpenFace: An open source facial behavior analysis toolkit', 2016 IEEE Winter Conference on Applications of Computer Vision (WACV), pp. 1-10.

Bertók, K. and Fazekas, A., 2016, Recognizing Complex Head Movements, Researchgate.Net.

Borghi, G. et al, 2018, Face-from-Depth for Head Pose Estimation on Depth Images, IEEE Transactions on Pattern Analysis and Machine Intelligence.

Bradski, G. and Kaehler, A., 2008, Learning OpenCV: Computer vision with the OpenCV library, O'Reilly Media, Inc.

Dalal, N. and Triggs, B., 2005, Histograms of Oriented Gradients for Human Detection, International Conference on Computer Vision \& Pattern Recognition (CVPR '05), pp. 886-893.

Eduardo Thomaz, C. and Antonio Giraldi, G., 2010, A new ranking method for principal components analysis and its application to face image analysis, Image and Vision Computing, pp. 902-913.

Fanelli, G. et al, 2011, Real time head pose estimation with random regression forests, CVPR 2011. IEEE, pp. 617-624.

Hearst, M. A. et al., 1998, Support vector machines, IEEE Intelligent Systems and their Applications, pp. 18-28.

King, D. E., 2009, Dlib-ml: A machine learning toolkit, Journal of Machine Learning Research, pp. 1755-1758.

Pedregosa, F. et al, 2011, Scikit-learn: Machine learning in Python, Journal of Machine Learning Research, pp. 2825-2830.

Peer, P. et al, 2014, Strategies for Exploiting Independent Cloud Implementations of Biometric Experts in Multibiometric Scenarios, Mathematical Problems in Engineering, pp. 1-15.

Ranjan, R., Patel, V. M. and Chellappa, R., 2017, HyperFace: A Deep Multi-Task Learning Framework for Face Detection, Landmark Localization, Pose Estimation, and Gender Recognition, IEEE Transactions on Pattern Analysis and Machine Intelligence, pp. 121-135.

Rekha, N. and Kurian, M., 2014, Face detection in real time based on HOG, International Journal of Advanced Research in Computer Engineering \& Technology (IJARCET), pp. 1345--1352.

Ruiz, N., Chong, E. and Rehg, J. M., 2018, Fine-Grained Head Pose Estimation Without Keypoints, Proceedings of the IEEE Conference on Computer Vision and Pattern Recognition Workshops, pp. 2074--2083.

Sagonas, C. et al, 2013, 300 Faces in-the-Wild Challenge: The first facial landmark localization Challenge, Proceedings of the IEEE International Conference on Computer Vision Workshops, pp. 397--403.

Valle, R. et al, 2016, Head-Pose Estimation In-the-Wild Using a Random Forest, International Conference on Articulated Motion and Deformable Objects. Springer, pp. 24-33.

Wang, X., Han, T. X. and Yan, S, 2009, An HOG-LBP human detector with partial occlusion handling, 2009 IEEE 12th International Conference on Computer Vision. IEEE, pp. 32-39.

Yang, T.-Y. et al, 2019, FSA-Net: Learning Fine-Grained Structure Aggregation for Head Pose Estimation from a Single Image, Proceedings of the IEEE Conference on Computer Vision and Pattern Recognition, pp. 1087--1096. 